\title{
Protective effect of the polarity of macrophages regulated by IL-37 on atherosclerosis
}

\author{
J. Huang', F.L. Hou ${ }^{2}$, A.Y. Zhang ${ }^{1}$ and Z.L. Li ${ }^{2}$ \\ ${ }^{1}$ Department of Cardiology, Weifang People's Hospital, Weifang, Shandong, \\ China \\ ${ }^{2}$ Department of Health, Weifang People's Hospital, Weifang, Shandong, China \\ Corresponding author: Z.L. Li \\ E-mail: zhilingdoctor8@163.com
}

Genet. Mol. Res. 15 (2): gmr.15027616

Received September 10, 2015

Accepted December 21, 2015

Published May 13, 2016

DOI http://dx.doi.org/10.4238/gmr.15027616

ABSTRACT. As an anti-inflammatory cytokine, interleukin-37 (IL37) provides certain protective effects against inflammatory and autoimmune diseases. Recent reports indicate that IL-37 is expressed in foam cells of atherosclerotic plaques in both the coronary and carotid arteries of humans, suggesting the possible involvement of IL-37 in the pathogenesis and progression of atherosclerosis. Current evidence supports the protective role that IL-37 plays against atherosclerosis via the regulation of different subtypes of macrophage. Atherosclerosis was induced in apolipoprotein E -/- mice through diet, and the mice were then given IL-37 to observe patterns in the aorta plaque. Furthermore, human peripheral blood-derived monocytes were cultured for seven days to induce the differentiation of macrophages. Specifically, we observed the effect of IL-37 on oxygenated low density lipoprotein (ox-LDL)-induced macrophage polarity, in addition to conducting an expressional assay of the M1 cell markers tumor necrosis factor (TNF)- $\alpha$ and CD86 and the M2 marker CD206. IL-37 effectively decreased the area ratio between the aorta plaque and vascular cavity. We also observed that M1 macrophages can be induced from peripheral monocytes by ox-LDL, with significant elevation of marker molecules 
TNF- $\alpha$ and CD86. The co-stimulation of IL-37 and ox-LDL, however, inhibited the induction of M1 cells and facilitated the transformation of macrophages into M2 cells, as supported by the elevation of cellspecific marker CD206. These results indicate that IL-37 can prevent atherosclerosis by modulating macrophage polarity.

Key words: IL-37; Macrophage polarity; Atherosclerosis; Artery plaque

\section{INTRODUCTION}

Atherosclerosis is a current major health concern and demonstrates an increasing occurrence rate and decreasing age of primary onset. The lack of effective treatments contributes to its high mortality and disability rates. Inflammation is a major risk factor in the occurrence and development of atherosclerosis. Recent studies have shown the important role of the transformation between M1 and M2 sub-type macrophages in atherosclerosis (Wu et al., 2013). Different macrophage sub-types can be observed in atherosclerotic plaques. As previously reported, about $40 \% \mathrm{M} 1$ cells and $20 \% \mathrm{M} 2$ cells reside in the plaques of model mice (Adamson and Leitinger, 2011). Oxygenated low density lipoprotein (ox-LDL) can facilitate the transformation of macrophage or M2 cells into M1 cells and stimulate the secretion of pro-inflammatory cytokines including interleukin-6 (IL-6), IL-18, and monocyte chemotactic factor-1, thereby accelerating the progression of atherosclerosis (Duewell et al., 2010; Hirose et al., 2011; van Tits et al., 2011). Therefore, the modulation between different subtypes of macrophage may provide new drug targets for the treatment of atherosclerosis.

IL-37 is a novel anti-inflammatory cytokine in the IL-1 family and exerts a negative modulatory role in the inflammatory response of innate immunity. Mainly present in the brain, kidney, heart, and monocytes or dendritic cells in peripheral blood, IL-37 has been widely reported to have anti-inflammatory and immune suppressing functions (Boraschi et al., 2011; Dinarello and Bufler, 2013; Teng et al., 2014). Studies have also indicated the role IL-37 plays in inhibiting the activation of macrophages and discovered its expression in the foam cells of atherosclerotic plaques in the coronary artery and carotid (Ji et al., 2014). These studies indicated a potential protective role of IL-37 against atherosclerosis. Therefore, we hypothesized that IL-37 may be able to inhibit the occurrence and progression of atherosclerosis by modulating the transformation of different subtypes of macrophage. In this study, we investigated if IL-37 can regulate the polarity of macrophages and inhibit the formation of atherosclerotic plaques.

\section{MATERIAL AND METHODS}

\section{Animal models}

Sixteen male apolipoprotein E knockout mice (8 weeks old, body weight 22-25 g, provided by Weitong Lihua Corp., China) were randomly divided into the control group $(\mathrm{N}=8)$ and IL-37 treatment group $(\mathrm{N}=8)$. After 8 weeks of high fat and high cholesterol diet feeding, treatment animals were intraperitoneally injected with $2 \mathrm{mg}$ recombinant IL37 protein (R\&D Corp., USA) daily for two weeks, while control mice were given $200 \mu \mathrm{L}$ saline for two weeks. 


\section{Sample collection and preparation}

All mice were perfused with saline and $4 \%$ paraformaldehyde and were then sacrificed for collection of the heart and aorta, which were then fixed in $4 \%$ paraformaldehyde after separating peripheral tissues. The heart was then dissected horizontally into two parts. The upper section was dehydrated, embedded in paraffin, and cut into consecutive slices 5 -mm thick. Six slices were chosen every $40 \mathrm{~mm}$ for further hematoxylin-eosin and oil red $\mathrm{O}$ staining. The tissue morphology of atherosclerotic plaques was observed under a light field microscope. The Image Pro Plus software package (Version 6.0 for Windows) was used to analyze tissue morphology for parameters including vessel cavity area, plaque are and plaque/cavity area ratio. Averaged values were obtained for further statistical analysis.

\section{Separation and induction of human macrophages}

Venous blood $(5 \mathrm{~mL})$ was collected from healthy donors in EDTA-treated tubes. Monocytes were separated using the density gradient centrifugation method and were resuspended in RPMI 1640 medium. Cells were cultured with $5 \% \mathrm{CO}_{2}$ for $60 \mathrm{~min}$ to collect adhesion cells. Giemsa staining confirmed the monocyte nature of such cells with purity $>90 \%$. Trypan blue staining revealed the cell viability was $>95 \%$. Collected monocytes were re-suspended in RPMI 1640 containing 20\% human serum. Cells at $1 \times 10^{6} / \mathrm{mL}$ were seeded on 24-well plates for further induction of macrophages in three groups: 1) control; 2) ox-LDL $(50 \mathrm{mg} / \mathrm{L})$, and 3) ox-LDL (50 mg/L) and IL-37 (50 mg/L). All cells were cultured for 7 days to induce the differentiation of macrophages.

\section{Western blotting for macrophage markers}

Cultured cells in all groups were lysed in RIPA lysing buffer (with 1\% PMSF) on ice for $30 \mathrm{~min}$, followed by centrifugation at 13,000 rpm for $30 \mathrm{~min}$. Supernatants were quantified for protein concentration. Equal amounts $(30 \mathrm{mg})$ were separated by SDS-PAGE and transferred to a PVDF membrane, which was then blocked in 5\% defatted milk powder for $1 \mathrm{~h}$. Rabbit antibody against tumor necrosis factor (TNF)- $\alpha$ (Cell Signaling, USA), CD68 (Cell Signaling, USA), CD206 (Abcam, USA), and $\beta$-actin (Cell Signaling, USA), all at 1:1000 dilutions, were added to incubate the membrane overnight. On the next day, the membrane was incubated with goat anti-rabbit IgG (Cell Signaling, USA), diluted 1:500, for $1 \mathrm{~h}$. Colors were developed using ECL reagents. Staining images were captured under a light-field microscope, and the Image J software was used to quantify the protein expression level based on the optical density of blotting bands.

\section{Statistical analysis}

The SPSS 13.0 software package was used to process all collected data, which were first tested for normality. Measurement data are reported as means \pm standard deviation. Between-group comparisons were performed by the Student $t$-test while the analysis among multiple groups was performed with a one-way analysis of variance (ANOVA) followed by the post-hoc SNK test. Results were considered statistically significance when $\mathrm{P}<0.05$. 


\section{RESULTS}

\section{IL-37 decreases the aorta plaque/vessel cavity area ratio of atherosclerotic mice}

Histological observations showed that IL-37 intervention effectively decreased the ratio of aorta plaque/vessel cavity area in atherosclerotic mice, suggesting an inhibitory role of IL-37 in the formation of atherosclerotic plaques (Table 1).

Table 1. Plaque area and vessel cavity area in atherosclerotic mice.

\begin{tabular}{l|c|c|c|c}
\hline Group & $\mathrm{N}$ & Cavity area $\left(\mu \mathrm{m}^{2}\right)$ & Plaque area $\left(\mu \mathrm{m}^{2}\right)$ & Plaque/cavity area ratio \\
\hline Control & 8 & $213.40 \pm 48.60$ & $168.20 \pm 60.82$ & $0.78 \pm 0.42$ \\
\hline IL-37 & 8 & $263.65 \pm 48.68$ & $30.13 \pm 10.75$ & $0.11 \pm 0.04^{*}$ \\
\hline
\end{tabular}

$* \mathrm{P}<0.05$ compared to control group.

\section{IL-37 modulates ox-LDL-induced macrophage polarity}

Western blot results (Figure 1) showed that ox-LDL induced the transformation of human peripheral blood-derived monocytes into primarily M1 type macrophages, with significant elevation in the protein expression of the major cell markers TNF- $\alpha$ and CD86. When co-applied with ox-LDL, IL-37 inhibited the transformation into M1 cells and facilitated the differentiation into M2 cells, as supported by an elevation in the major cell marker CD206.

A

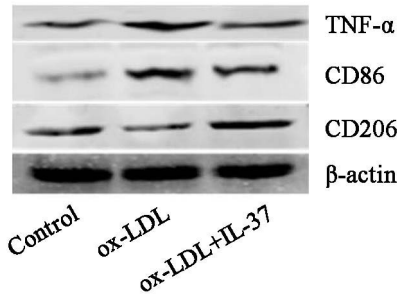

B

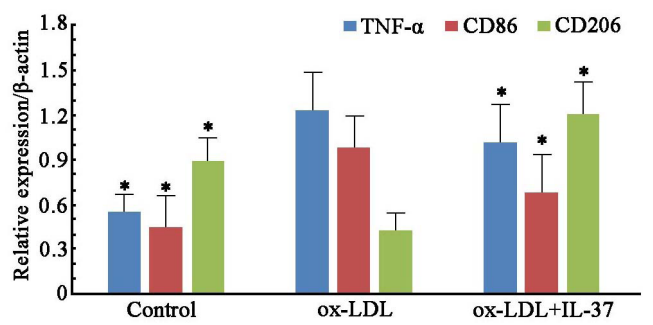

Figure 1. Effects of IL-37 on ox-LDL-induced macrophage polarity. A. Representative bands from western blotting. B. Quantified results showing relative protein expression levels. $* \mathrm{P}<0.05$ compared to ox-LDL group.

\section{DISCUSSION}

As an important component of the body's immune system, macrophages are believed to have pleiotropic roles, such as in immune induction and lipid phagocytes. They thereby play an important role in the pathogenesis and progression of atherosclerosis by facilitating inflammation and lipid deposition (Trpkovic et al., 2015; Lundqvist et al., 2015). Macrophages 
can differentiate into the M1 and M2 types, which can be inter-transformed depending on the body's microenvironment (Medbury et al., 2014; Chen et al., 2015). In atherosclerotic plaques, both M1 and M2 macrophages exist, although they are responsible for distinct functions (Fadini et al., 2014). These macrophages can be distinguished by the differential expression of cytokines, surface molecules, and arginine metabolic pathways (Peled and Fisher, 2014). Under the stimulation of lipoprotein, macrophages can differentiate into M1 cells, which induce the secretion of large amounts of pro-inflammatory factors including IL-12, IL-23, IL-6, and TNF- $\alpha$, all of which can participate in the inflammatory response and facilitate reactive oxygen release, thereby facilitating the progression of atherosclerosis (Singla et al., 2014). M2 cells, on the other hand, can be induced from macrophages under the direction of IL-4 and IL-13. M2 cells can inhibit the inflammatory response and facilitate the recovery of tissue injuries via the release of IL-10, arginase, and the scavenger receptor (Spivia et al., 2014). It is interesting to note that the major component of atherosclerotic plaques, oxygenated cholesterol, primarily facilitates the differentiation of M1 cells through activation of the MAP signaling pathway (Fang et al., 2010). All of these studies support a critical role of M1/M2 cell differentiation in the pathogenesis and progression of atherosclerosis.

As a newly identified member of the IL-1 family, IL-37 has been shown to have antiinflammatory and immune suppressing functions (Boraschi et al., 2011), and it is known to be involved in the progression of various inflammatory diseases (Nold et al., 2010). Current studies have demonstrated that such functions of IL-37 are mainly related to its inhibition of the release of inflammatory cytokines, including IL-1 $\beta$, IL-18, and TNF- $\alpha$ (Li et al., 2015; Nold-Petry et al., 2015). For example, IL-37 can potentiate the inhibitory function of IL-18BP on IL-18 via specific binding (Bufler et al., 2004). It is worth noting that recent studies have identified IL-37 expression in foam cells of atherosclerotic plaques in the human coronary artery and carotid (Wu et al., 2013; Ji et al., 2014), suggesting the potential role of IL-37 in the pathology of atherosclerosis. The nature of this function, i.e, whether a protective or aggravating role, has not been illustrated so far. Due to its anti-inflammatory nature, IL-37 was hypothesized in this study to play a protective role against atherosclerosis. Our in vitro studies showed that ox-LDL induced peripheral blood-derived monocytes to differentiate into M1 cells, as supported by the significant up-regulation of the M1 cell markers TNF- $\alpha$ and CD86. The co-stimulation of ox-LDL and IL-37, however, induced the macrophages to instead differentiate into M2 cells, with a corresponding elevation in CD206 protein expression. These results suggest that IL-37 can modulate the response of macrophages to liposomes and facilitate the differentiation towards anti-inflammatory M2 cells. Such results were also confirmed in atherosclerotic mice, in which recombinant IL-37 protein injection effectively inhibited the formation of atherosclerosis.

In summary, this study has demonstrated the modulatory role of IL-37 on macrophage

polarity, along with its protective function against atherosclerosis. The critical role of macrophages in atherosclerosis suggests that the transformation between different subtypes of macrophages may provide novel drug targets in treating atherosclerosis.

\section{Conflicts of interest}

The authors declare no conflict of interest. 


\section{ACKNOWLEDGMENTS}

We thank the anonymous reviewers for reviewing this manuscript.

\section{REFERENCES}

Adamson S and Leitinger N (2011). Phenotypic modulation of macrophages in response to plaque lipids. Curr. Opin. Lipidol. 22: 335-342. http://dx.doi.org/10.1097/MOL.0b013e32834a97e4

Boraschi D, Lucchesi D, Hainzl S, Leitner M, et al. (2011). IL-37: a new anti-inflammatory cytokine of the IL-1 family. Eur. Cytokine Netw. 22: 127-147.

Bufler P, Gamboni-Robertson F, Azam T, Kim SH, et al. (2004). Interleukin-1 homologues IL-1F7b and IL-18 contain functional mRNA instability elements within the coding region responsive to lipopolysaccharide. Biochem. J. 381 : 503-510. http://dx.doi.org/10.1042/BJ20040217

Chen KS, Chen PN, Hsieh YS, Lin CY, et al. (2015). Capsaicin protects endothelial cells and macrophage against oxidized low-density lipoprotein-induced injury by direct antioxidant action. Chem. Biol. Interact. 228: 35-45. http://dx.doi. org/10.1016/j.cbi.2015.01.007

Dinarello CA and Bufler P (2013). Interleukin-37. Semin. Immunol. 25: 466-468. http://dx.doi.org/10.1016/j. smim.2013.10.004

Duewell P, Kono H, Rayner KJ, Sirois CM, et al. (2010). NLRP3 inflammasomes are required for atherogenesis and activated by cholesterol crystals. Nature 464: 1357-1361. http://dx.doi.org/10.1038/nature08938

Fadini GP, Simoni F, Cappellari R, Vitturi N, et al. (2014). Pro-inflammatory monocyte-macrophage polarization imbalance in human hypercholesterolemia and atherosclerosis. Atherosclerosis 237: 805-808. http://dx.doi. org/10.1016/j.atherosclerosis.2014.10.106

Fang L, Harkewicz R, Hartvigsen K, Wiesner P, et al. (2010). Oxidized cholesteryl esters and phospholipids in zebrafish larvae fed a high cholesterol diet: macrophage binding and activation. J. Biol. Chem. 285: 32343-32351. http:// dx.doi.org/10.1074/jbc.M110.137257

Hirose K, Iwabuchi K, Shimada K, Kiyanagi T, et al. (2011). Different responses to oxidized low-density lipoproteins in human polarized macrophages. Lipids Health Dis. 10: 1. http://dx.doi.org/10.1186/1476-511X-10-1

Ji Q, Zeng Q, Huang Y, Shi Y, et al. (2014). Elevated plasma IL-37, IL-18, and IL-18BP concentrations in patients with acute coronary syndrome. Mediators Inflamm. 2014: 165742. http://dx.doi.org/10.1155/2014/165742

Li S, Neff CP, Barber K, Hong J, et al. (2015). Extracellular forms of IL-37 inhibit innate inflammation in vitro and in vivo but require the IL-1 family decoy receptor IL-1R8. Proc. Natl. Acad. Sci. USA 112: 2497-2502. http://dx.doi. org/10.1073/pnas.1424626112

Lundqvist A, Magnusson LU, Ullström C, Krasilnikova J, et al. (2015). Oregonin reduces lipid accumulation and proinflammatory responses in primary human macrophages. Biochem. Biophys. Res. Commun. 458: 693-699. http:// dx.doi.org/10.1016/j.bbrc.2015.01.161

Medbury HJ, Williams H and Fletcher JP (2014). Clinical significance of macrophage phenotypes in cardiovascular disease. Clin. Transl. Med. 3: 63.http://dx.doi.org/10.1186/s40169-014-0042-1

Nold MF, Nold-Petry CA, Zepp JA, Palmer BE, et al. (2010). IL-37 is a fundamental inhibitor of innate immunity. Nat. Immunol. 11: 1014-1022. http://dx.doi.org/10.1038/ni.1944

Nold-Petry CA, Lo CY, Rudloff I, Elgass KD, et al. (2015). IL-37 requires the receptors IL-18R $\alpha$ and IL-1R8 (SIGIRR) to carry out its multifaceted anti-inflammatory program upon innate signal transduction. Nat. Immunol. 16: 354-365. http://dx.doi.org/10.1038/ni.3103

Peled M and Fisher EA (2014). Dynamic aspects of macrophage polarization during atherosclerosis progression and regression. Front. Immunol. 5: 579. http://dx.doi.org/10.3389/fimmu.2014.00579

Singla RD, Wang J and Singla DK (2014). Regulation of Notch 1 signaling in THP-1 cells enhances M2 macrophage differentiation. Am.J.Physiol. HeartCirc. Physiol.307:H1634-H1642.http://dx.doi.org/10.1152/ajpheart.00896.2013

Spivia W, Magno PS, Le P and Fraser DA (2014). Complement protein C1q promotes macrophage anti-inflammatory M2-like polarization during the clearance of atherogenic lipoproteins. Inflamm. Res. 63: 885-893. http://dx.doi. org/10.1007/s00011-014-0762-0

Teng X, Hu Z, Wei X, Wang Z, et al. (2014). IL-37 ameliorates the inflammatory process in psoriasis by suppressing proinflammatory cytokine production. J. Immunol. 192: 1815-1823. http://dx.doi.org/10.4049/jimmunol.1300047

Trpkovic A, Resanovic I, Stanimirovic J, Radak D, et al. (2015). Oxidized low-density lipoprotein as a biomarker of cardiovascular diseases. Crit. Rev. Clin. Lab. Sci. 52: 70-85.

van Tits LJ, Stienstra R, van Lent PL, Netea MG, et al. (2011). Oxidized LDL enhances pro-inflammatory responses of alternatively activated M2 macrophages: a crucial role for Krüppel-like factor 2. Atherosclerosis 214: 345-349. http://dx.doi.org/10.1016/j.atherosclerosis.2010.11.018

Wu BW, Zeng QT, Meng K and Ji QW (2013). The potential role of IL-37 in atherosclerosis. Pharmazie 68: 857-860.

Genetics and Molecular Research 15 (2): gmr.15027616

CFUNPEC-RP www.funpecrp.com.br 